\title{
Research on Vehicle Detection Algorithm based on Haar-like and CNN
}

\author{
Jinlong Chen ${ }^{1}$, Yiming Jiang ${ }^{2}$ and Minghao Yang ${ }^{1+}$ \\ ${ }^{1}$ Guangxi Key Laboratory of Cryptography and Information Security , Guilin University of Electronic \\ Technology, China \\ ${ }^{2}$ Guangxi Key Laboratory of Trusted Software, Guilin University of Electronic Technology, China
}

\begin{abstract}
Vehicle detection algorithms exists in various application scenarios, including driverless car and traffic control. We proposed a vehicle detection method based on Haar-like and Convolutional Neural Networks to address the problems of traditional Adaboost algorithm for Haar features has low precision when detecting vehicles. In this paper we proposed a method which through the Haar-like and integral maps to extract target features, calculate eigenvalues, then uses Convolutional Neural Networks to discriminate and remove false targets. Experimental results prove: our algorithm proposed in this paper can effectively improve the precision of detection at a good recognition speed.
\end{abstract}

Keywords: Vehicle detection, Haar-like feature, CNN, Adaboost algorithm.

\section{Introduction}

Vehicle detection is a research hotspot in the field of computer vision. Vehicle detection is a prerequisite for road management and unmanned driving. With the development of the economy, motor vehicles continue to enter millions of households. With the rapid growth of car ownership, the pressure on traffic management is also increasing. How to quickly detect vehicles in urban traffic and highways has become a key technology of intelligent traffic control systems.

At present, the more commonly used vehicle detection methods are to detect by toroidal coils, lidar, infrared, pneumatic catheters, vision sensors and other equipment and instruments, among them, the visual sensor has become a mainstream method in vehicle detection due to its advantages such as low price, simple method, abundant information reception, and non-contact measurement [1].

Haar-like is a classic image feature extraction algorithm. It was first used by Papageorigiou et al. for face description [2]. In recent years, there have been continuous improvements to the traditional Haar features to further improve the detection effect. Roxana et al. [3] used a class of support vector machine (OC-SVM) classification to detect moving vehicles. This method first uses an adaptive hybrid Gaussian model to detect the vehicle area, extracts geometric features, and uses Kalman estimation to track. However, this method has weak real-time performance and average detection effect. Daniel et al. [4] proposed an image classifier method based on the combination of Haar-like, LBP, and HOG features, using a stereo model to detect moving vehicles, but this method has a complicated algorithm and poor detection efficiency, and is only suitable for specific environments.

Convolutional Neural Networks is a kind of Artificial Neural Network. CNN uses a weight-sharing strategy to reduce parameters and has a high degree of invariance to the translation, zoom, tilt, or other forms of deformation of the image, so it is widely used in the detection and recognition of two-dimensional image targets. CNN can abstract features at different levels in different stages of learning, avoiding the process of

\footnotetext{
+ Corresponding author. Tel.: +86 15811155263.

E-mail address: 648666565@qq.com.
} 
manually designing features and classifiers in traditional machine learning algorithms. Recently, CNN and its derivative algorithms have developed rapidly in image recognition, and deep learning-based object detection algorithms have made breakthrough progress, mainly divided into two categories. One type is use region proposal represented by R-CNN [5] and Faster R-CNN [6]; the other is a regression-based method represented by YOLO [7] and SSD. In terms of vehicle detection, Linhui Li et al. [8] proposed a method of segmenting the bottom shadow of the vehicle first, and then training the convolutional neural network to filter out false detections to detect the vehicle. Karaimer et al. [9] proposed a vehicle detection method according to the shape (contour) of a moving object obtained by background subtraction and using multiple consecutive frames to eliminate false discriminations. However, the above methods mainly have the problems of complex training process, manual optimization of a large number of hyperparameters and slow detection speed.

In this paper, we introduce Convolutional Neural Networks to make a second decision on the targets detected by Haar-like features, and then get the final result. Compared with the traditional Haar-like detection, the method employed in this paper reach a certain improvement in detection precision. Compared with the detection algorithm using convolutional neural network directly, the vehicle detection algorithm based on Haar-like and CNN proposed in this paper greatly reduces the detection time, under the premise of sacrifice a small amount of calculation time, the accuracy of vehicle detection is guaranteed.

\section{Detection Algorithm Based on Haar-like Features and CNN}

\subsection{Haar-like Features}

The Haar-like classifier detects targets by using the characteristics of known training samples and comparing them with the characteristics of unknown images to achieve target detection. Haar-like detection first extracts features from training samples, then calculates feature values and builds a weak classifier, and finally constructs it into a strong classifier. Traditional Haar-like features include edge features, linear features, central features, and diagonal features. However, for targets such as vehicles with randomness, large grayscale image differences, and small background grayscale differences, traditional calculation methods cause poor detection performance of weak classifiers, which makes the strong classifier need a large number of weak classifiers when cascading. Therefore, this paper uses the extended Haar-like feature to divide the sum of pixels in black and white areas to enhance the performance of weak classifier detection. The feature map is shown in Figure 1.

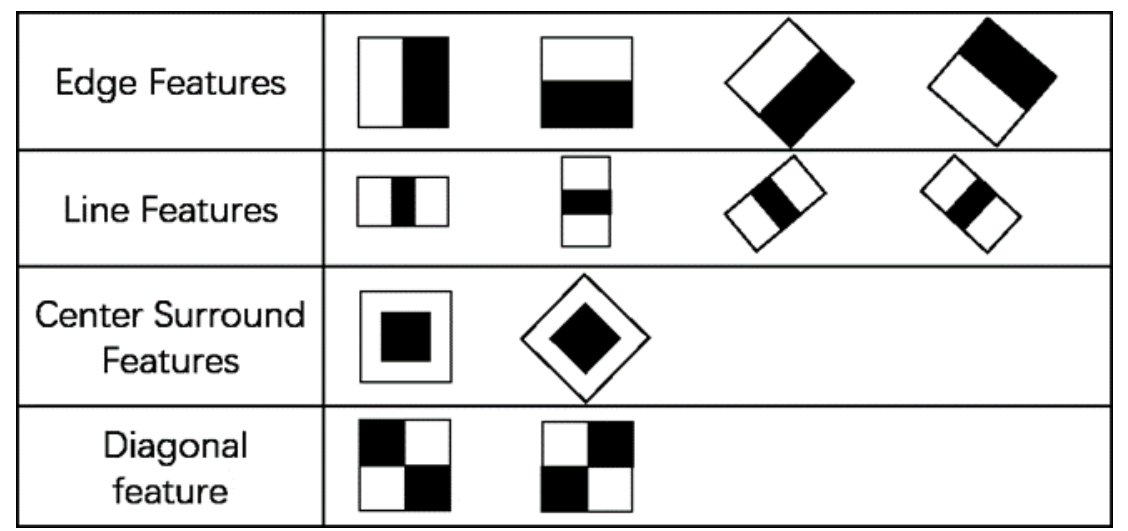

Fig. 1: Haar-like feature.

\subsection{Integral Graph}

After obtaining the rectangular features, in order to quickly calculate the values of the rectangular features. VIOLA et al. [10] proposed a method for obtaining features values using integral graphs. The integral diagram is shown in Figure 2. The integral map only needs to traverse the image once to find any coordinate point $(\mathrm{x}, \mathrm{y})$ in the upper left corner of the image.

$$
\mathrm{ii}(\mathrm{x}, \mathrm{y})=\sum_{x^{\prime} \ll x, y^{\prime} \ll y} i\left(x^{\prime}, y^{\prime}\right)
$$

Where: $\mathrm{ii}(\mathrm{x}, \mathrm{y})$ is the integral graph and $\mathrm{i}(\mathrm{x}, \mathrm{y})$ is the original image. 
In Figure 2: $A(x, y)$ is the integral graph of point $(x, y) ; s(x, y)$ is the sum of all original image pixels in the $\mathrm{y}$ direction of point $(\mathrm{x}, \mathrm{y})$. Its expression is as follows:

$$
\begin{aligned}
\mathrm{s}(\mathrm{x}, \mathrm{y}) & =s(x, y-1)+i(x, y) \\
\mathrm{ii}(\mathrm{x}, \mathrm{y}) & =i i(x-1, y)+s(x, y)
\end{aligned}
$$

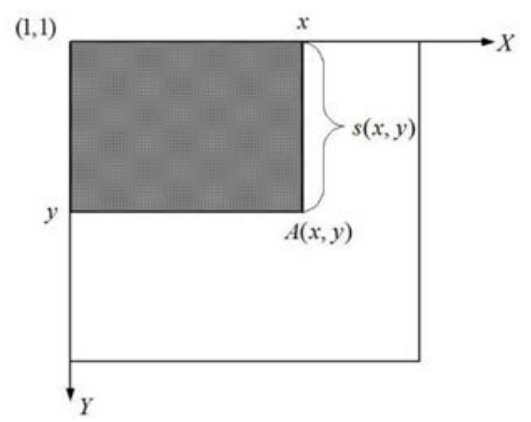

Fig. 2: Integral diagram of point coordinates.

\subsection{Feature Extraction Based on Adaboost}

\subsubsection{Weak Classifier}

The weak classifier calculates only the local feature values of the input image to determine whether the input image is the target. The mathematical expression of the weak classifier is:

$$
\mathrm{h}(\mathrm{x}, \mathrm{f}, \mathrm{p}, \theta)=\left\{\begin{array}{lr}
1 & f(x)<p \theta \\
0 & \text { other }
\end{array}\right.
$$

Where: $\mathrm{f}$ is the feature, $\theta$ is the threshold, $\mathrm{p}$ indicates the direction of the inequality sign, $\mathrm{x}$ represents a detection subwindow. For each feature $f$, training a weak classifier $h(x, f, p, \theta)$ is to determine the optimal threshold value of $f$ and make this weak classifier $h(x, f, p, \theta)$ have the lowest classification error on all training samples.

The process of weak classifier training is roughly divided into the following steps:

1. For each feature $\mathrm{f}$, calculate the feature values of all training samples.

2. Sort the feature values.

3. For each element that is sorted, calculate the sum of weights of all positive and negative examples $\mathrm{T}+, \mathrm{T}-$, and the sum of weights of positive and negative examples before the element $\mathrm{S}+, \mathrm{S}-$.

4. Select the feature values $F_{k_{i}}$ of the current element and the number between the eigenvalue $F_{k_{i-1}}$ in front of it as the threshold. The classification error of this threshold is:

$$
\mathrm{e}=\min \left(S^{+}+\left(T^{-}-S^{-}\right), S^{-}+\left(T^{+}-S^{+}\right)\right)
$$

\subsubsection{Strong Classifier}

Multiple weak classifiers form a strong classifier. The strong classifier can better process the object. By cascading the trained multiple strong classifiers, it can quickly detect the target from the target image.

The training process of the strong classifier is shown below.

1. Given a series of training samples $\left(x_{1}, y_{1}\right),\left(x_{2}, y_{2}\right), \ldots \ldots\left(x_{n}, y_{n}\right)$, where $y_{i}=0$ is a negative sample, $y i=1$ is a positive sample, and $\mathrm{n}$ is a total of training samples.

2. Initial $\mathrm{D}(\mathrm{i})=1 / \mathrm{n}$.

3. For $\mathrm{t}=1,2, \ldots \mathrm{T}$ :

- Weight normalization:

$$
w_{t, i}=\frac{w_{t, i}}{\sum_{j-1}^{n} w_{t, i}}
$$

- For each feature $f_{j}$ train a weak classifier $h_{j}$, each classifier is trained using only one Haar-like feature. Classification error is

$$
\varepsilon_{j}=\sum_{i} w_{i}\left|h_{j}\left(x_{i}\right)-y_{i}\right|
$$

- Find a weak classifier $h j$ with the smallest classification error from the weak classifiers

determined in the previous step. 
- $\quad$ Update the weight of each sample:

$$
w_{t+1, i}=w_{t, i} \beta_{t}^{1-e_{i}}
$$

- The resulting strong classifier consists of:

\subsection{VD-CNN Network Structure}

$$
\mathrm{h}(\mathrm{x})=\left\{\begin{array}{rr}
1 \sum_{t-1}^{T} \alpha_{t} h_{t}(x) \geq \frac{1}{2} \sum_{t=1}^{T} \alpha_{t} \\
\text { otherwise }
\end{array}\right.
$$

We proposed a convolutional neural network, VDNet, to improve the precision of HAAR-LIKE feature detection and the existence of a large number of false targets. The target area identified by Haar-like feature detection is put into the CNN network for secondary judgment, so as to obtain more accurate results. This network uses 3 convolution layers and 2 pooling layers. The input data is a gray-scaled image with a size of $30 \times 30$ pixels. The size of the convolution kernel in the first convolution layer is $3 \times 3$, a total of 32 convolution kernels, and the convolution output is 32 feature maps of $28 \times 28$; The first pooling layer uses maximum pooling, with a size of $2 \times 2$, a step size of 2 , and an output matrix of $14 \times 14 \times 32$; The size of the convolution kernel in the second convolution layer is $3 \times 3$, a total of 64 convolution kernels, and the convolution output is 64 feature maps of $12 \times 12$; The size of the convolution kernel in the third convolution layer is $3 \times 3$, a total of 64 convolution kernels, and the convolution output is 64 feature maps of $10 \times 10$; The second pooling layer uses maximum pooling, with a size of $2 \times 2$, a step size of 2 , and an output matrix of $5 \times 5 \times 64$; Finally, the results of the target are obtained through the outputs of 3 FC layers and 2 sigmoid classifiers. The network structure of VD-CNN is shown in Figure 3 below.

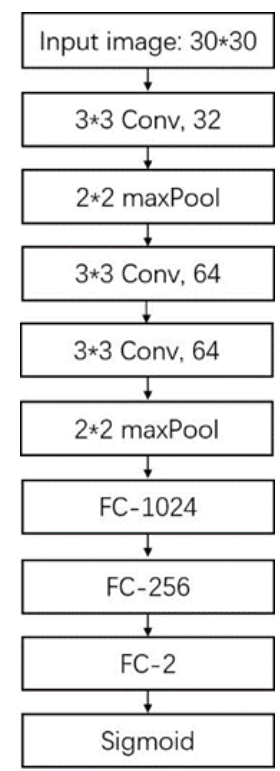

Fig. 3: VD-CNN Network Structure

\section{Experimental Design and Results Analysis}

\subsection{Experimental Data}

In this paper, Hangzhou, Guilin and Beijing are selected as three cities, and 5 intersections are randomly selected for video data collection in each city. In order to make the detection effect more accurate, this paper selects vehicle data from the MIT CBCL data set to expand the training samples and merges them into positive samples of the training set. The positive samples include front and rear views of the vehicle, 45degree views and side views. Negative samples consist of road information and surrounding buildings that do not include vehicles.

\subsection{Data Preprocessing}

1. Perform grayscale processing on the acquired samples, convert color samples into grayscale pictures.

2. In Haar-like feature training, normalize the positive samples to $20 \times 20$ pixels, and randomly normalize the negative samples to between $60 \times 60$ pixels and $144 \times 144$ pixels. 
3. In CNN training, the positive and negative samples are uniformly normalized to $30 \times 30$ pixels, and the negative samples include the wrong targets marked during Haar-like detection.

After pretreatment, the number of positive samples for Haar-like training is 12,000 and the number of negative samples is 24,000 . In CNN training, the number of positive samples is 10,000 and the number of negative samples is 11,000. The original samples and the pretreated samples are shown in Figure 4 below.

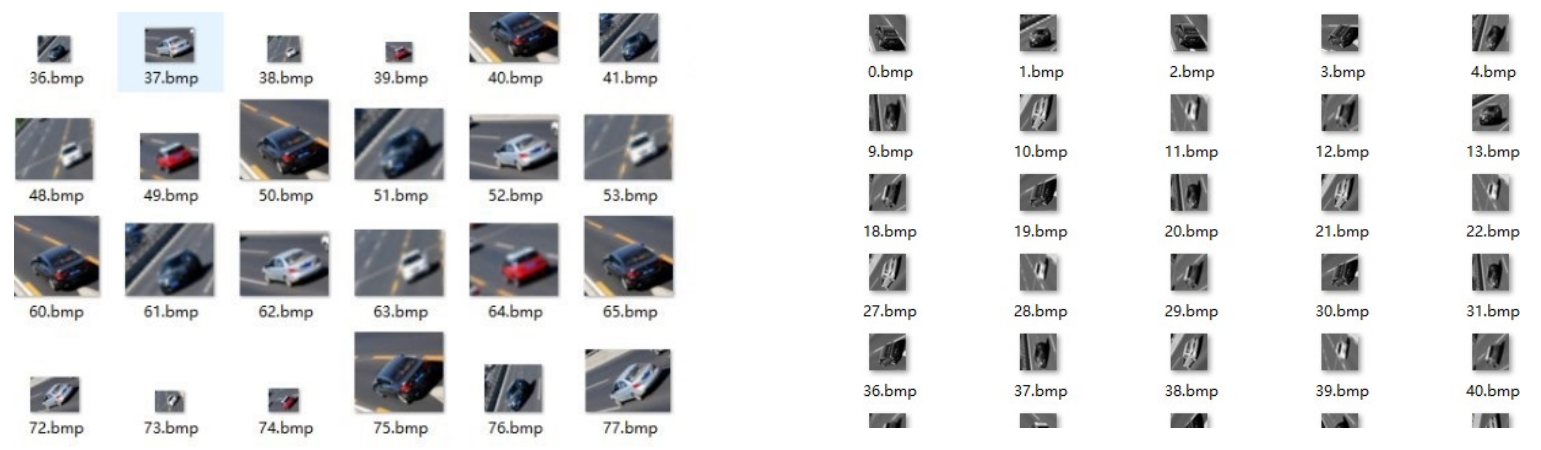

Fig. 4: Original Samples and Preprocessed Samples

\subsection{Evaluation Index}

In order to accurately detect the effectiveness of Haar-like and CNN-based vehicle detection algorithms, this article uses the following indicators to evaluate the performance of the algorithm based on commonly used target detection performance evaluation indicators and evaluation indicators used in most target detection algorithms:

- Precision

$$
\text { Precision }=\frac{T P}{T P+F P}
$$

Where: TP is the number of correctly classified as positive examples, FP is the number of incorrectly classified as positive examples.

- Recall

$$
\text { Recall }=\frac{T P}{T P+F N}
$$

Where: TP is the number of correctly classified as positive examples, FN is the number of incorrectly classified as negative examples.

- Average Precision, AP

The Average Precision is the average of the precision of each point of Recall from 0 to 1 .

$$
\mathrm{AP}=\int_{0}^{1} p(r) d r
$$

Where: $\mathrm{p}$ is Precision, $\mathrm{r}$ is Recall, $\mathrm{p}$ is a function that takes $\mathrm{r}$ as a parameter.

\subsection{Results Analysis}

Our experimental results are shown in Table 1. The table gives the precision, recall, average accuracy, mean average precision and speed five indicators to evaluate the experimental results. According to the experimental results, it can be seen that the vehicle detection algorithm based on Haar-like features and CNN has improved on these indicators. The size of the experimental input image is $608 * 608$ pixels. The speed in the table below is the detection speed for a single image.

Table 1. Experimental Result

\begin{tabular}{|c|c|c|c|c|}
\hline Method & Precision & Recall & AP & SPEED \\
\hline Standard Haar & 0.7708 & 0.8803 & 0.6687 & $80 \mathrm{~ms}$ \\
\hline Improved Haar [11] & 0.8163 & 0.8989 & 0.7315 & $110 \mathrm{~ms}$ \\
\hline Haar SVM [12] & 0.8811 & 0.8925 & 0.8489 & $320 \mathrm{~ms}$ \\
\hline YOLOV3-TINY & 0.9281 & 0.9078 & 0.8489 & $630 \mathrm{~ms}$ \\
\hline HaarCNN(OURS) & 0.9233 & 0.8975 & 0.8374 & $270 \mathrm{~ms}$ \\
\hline
\end{tabular}


By comparing the results of the three algorithms Standard Haar, Improved Haar and HaarCNN, we can see that the Improved Haar feature detection algorithm can get more vehicle features than Standard Haar feature detection algorithm, thereby increasing the number of true positive of detected. However, the improvement effect of this method is not obvious, and the recognition rate of the wrong target is still very low. By comparing the results of the HaarSVM method and the HaarCNN method proposed in this paper, we can know that the convolutional neural network has a better discrimination effect than SVM. Compared with the YOLOv3-TINY algorithm that directly uses CNN, YOLOv3-TINY has a small number of advantages in the detection effect. However, in terms of detection speed, the calculation time required by the convolutional neural network is large, and for general hardware devices, real-time detection effects cannot be achieved.

Therefore, the vehicle detection algorithm employed in this paper can effectively detect the vehicle, and effectively eliminate the virtual scene problem in the traditional haar- like detection without increasing the calculation time.

\section{Conclusion}

This paper proposes a vehicle detection method based on Haar-like features and CNN. This algorithm introduces the Convolutional Neural Networks to perform secondary discrimination on Haar-like features detection, thereby improving the problem of low precision when using traditional Haar-like feature detection. Through real-time detection and verification on video data in 15 intersections from 3 cities. Our experimental results show that compared with the original HAAR-LIKE feature detection, the algorithm employed in this paper has significantly improved the accuracy of vehicle detection. Compared with the detection algorithm using convolutional neural network directly, the proposed algorithm has certain advantages in detection speed under the premise of ensuring detection precision. Future work includes: optimizing the network structure of the CNN model to further improve the precision and detect speed.

\section{Acknowledgements}

This research work is supported by the grant of Guangxi science and technology development project (No: AB17195027), the grant of Guangxi scientific research and technology development plan project (No: AB18221011), the grant of Guangxi Key Laboratory of Trusted Software of Guilin University of Electronic Technology (No: KX201620), the grant of Guilin science and technology project Research and application demonstration of key technologies of somatosensory interaction based on gesture (No: 20180107-4).

\section{References}

[1] XU, GAO F, XU G Y. An algorithm for front-vehicle detection based on Haar - like feature [J]. // Automotive Engineering, 2013, 35 (4): 381 - 384.

[2] IZADINIA H, RAMAK RISHNA V, KITANI KM, et al. Multi-pose multi-target tracking for activity understanding [C] // Proceedings of Applications of Computer Vision (WACV). Tampa: IEEE, 2013.

[3] Roxana V-P, Alberto S-R, Deni T-R, et al. Vehicle Detection with Occlusion Handling, Tracking, and OC-SVM Classification: A High Performance Vision-Based System [J]. // Sensors, 2018, 18 (2): 374.

[4] Daniel N, Tobias L, Fritz U, et al. Online vehicle detection using Haar-like, LBP and HOG feature based image classifiers with stereo vision preselection [C] // IV2017: 2017 Intelligent Vehicles Symposium. USA: IEEE, 2017: 773-778.

[5] Girshick R, Donahue J, Darrell T, et al. Region based convolutional networks for accurate object detection and segmentation[J]. // IEEE Transactions on Pattern Analysis and Machine Intelligence, 2016, 38(1): 142-158.

[6] Ren S Q, He K M, Girshick R, et al. Faster RCNN: towards real-time object detection with region proposal networks[J]. // IEEE Transactions on Pattern Analysis and Machine Intelligence, 2017, 39(6): 1137-1149.

[7] Redmon J, Divvala S, Girshick R, et al. You only look once: unified, real-time object detection[C] // IEEE Computer Vision and Pattern Recognition, Las Vegas, Nevada,2016:779-788.

[8] Li Lin-hui, Lun Zhimei, Lian Jing, et al. Convolution neural network-based vehicle detection method [J]. // Journal of Jilin University (Engineering and TechnologyEdition), 2017, 47 (2): 384-391. 
[9] Karaimer H C, Baris I, Bastanlar Y. Detection and classification of vehicles from omnidirectional videos using multiple silhouettes [J]. // Pattern Analysis and Applications, 2017, 20 (3): 893-905.

[10] VIOLA P, JONES M J. Rapid object detection using a boosted cascade of simple features [C] // Proceedings of the 2001 IEEE Computer Society Conference on Computer Vision and Pattern Recognition. CVPR, 2001.

[11] Pengpeng Ni, Haiquan Gu, Fengge Dong, Wenbin Wang. Research on Vehicle Detection Algorithm Based on Haar-like and Adaboost [J] // Auto Parts, 2019 (10): 5-9.

[12] Li Zhang. Pedestrian detection method based on SVM-AdaBoost algorithm [J]. // Industrial Instrumentation and Automation, 2016 (04): 117-120. 